\title{
Germanica
}

\section{Der „Popstar des Literaturbetriebes“ ist tot. Marcel Reich-Ranicki in Nachruf-Texten deutschsprachiger Tages- und Wochenzeitungen}

Ursula KLINGENBÖCK : "La pop star de la scène littéraire » est morte. Marcel Reich-Ranicki dans les nécrologies des quotidiens et hebdomadaires de langue allemande

Ursula KLINGENBÖCK: The "Pop Star of Literarure" is Dead. Obituaries on Marcel Reich-Ranicki in German-Speaking Daily and Weekly Press

\section{Ursula Klingenböck}

\section{OpenEdition}

\section{Journals}

Édition électronique

URL : https://journals.openedition.org/germanica/7998

DOI : 10.4000/germanica.7998

ISSN : 2107-0784

Éditeur

Université de Lille

Édition imprimée

Date de publication : 1 décembre 2019

Pagination : 111-126

ISBN : 978-2-913857-44-5

ISSN : 0984-2632

Référence électronique

Ursula Klingenböck, „Der „Popstar des Literaturbetriebes“ ist tot. Marcel Reich-Ranicki in NachrufTexten deutschsprachiger Tages- und Wochenzeitungen“, Germanica [Online], 65 | 2019, Online erschienen am: 01 Januar 2022, abgerufen am 08 Januar 2022. URL: http://journals.openedition.org/ germanica/7998; DOI: https://doi.org/10.4000/germanica.7998 


\title{
Der „Popstar des Literaturbetriebes“ ist tot. Marcel Reich-Ranicki in Nachruf-Texten deutschsprachiger Tages- und Wochenzeitungen
}

\author{
Ursula Klingenböck \\ Universität Wien
}

\section{Untersuchungsgegenstand, Fragestellung und Methodenreflexion}

Ingeborg Bachmann, Erich Fried, Ernst Jandl, Uwe Johnson, Wolfgang Koeppen, Anna Seghers und Peter Weiss gehören zu jenen, auf die Marcel Reich-Ranicki - häufig unter der Überschrift „Zum Tode von..." - einen Nachruf verfasst hat. Im Zentrum des folgenden Beitrags steht allerdings nicht Reich-Ranicki als ,Nachrufer', sondern als derjenige, dem anlässlich seines Todes am 18.09.2013 - und durchaus in wörtlichem Sinn - etwas nachgesagt wird: unter anderem eine in das Bild des „Popstars“1 gefasste Popularität.

Die folgenden Überlegungen werden durch mehrere Wissenschaften angeleitet: von Textlinguistik und -pragmatik - dementsprechend wird der Kasualtext ,Nachruf' als Textsorte aufgefasst ${ }^{2}$; von Medienwissenschaften,

1. - Peter Schneider, „Marcel Reich-Ranicki wird 80. Eine vertrackte Liebesgeschichte“, Tagesspiegel, 01.06.2000.

2. - Eine (auch) poetische Qualität, wie sie als Distinktionsmerkmal der ,Gattung“ 
indem die Kommunikation als Ziel des Sprach- bzw. Texthandelns wesentlich durch das Medium (hier: das Massenmedium der Zeitung) konfiguriert wird; von der Soziologie, weil das Nachrufen als Handeln gedacht wird, das zwischen Akteuren (Subjekten, Institutionen, Organen) vonstattengeht; von den Diskurswissenschaften, indem der Nachruf als Teil des Totendiskurses verstanden werden kann, und nicht zuletzt von den Kulturwissenschaften, insofern sich die Praktik des Nachrufens innerhalb einer stark konventionalisierten kulturellen Praxis um Tod und Erinnerung verortet.

Der erste Teil des Beitrags sondiert Nachruftexte auf Marcel ReichRanicki und korreliert sie mit ihrer Redesituation bzw. den Medien ihrer Publikation. Der darauffolgende analytische Teil untersucht ein repräsentatives Textkorpus nach textlinguistischen (inhaltliche Akzentuierung, Umfang, Aufbau, Elokution und Einsatz affektstimulierender rhetorischer Mittel) ${ }^{3}$ und -pragmatischen (Sprachbzw. Texthandlungen in ihren medialen und situativen Kontexten) ${ }^{4}$ Parametern. Das Erkenntnisinteresse ist dabei im Unterschied zu Referenzstudien ${ }^{5}$ weder historisch noch systemisch ausgerichtet; was die Analyse exemplarischer Nachrufe aber entwerfen kann und soll, sind Konturen ,des ${ }^{6}$ Nachrufs auf Reich-Ranicki in ausgewählten Tages- und Wochenzeitungen. Ein wesentlicher Aspekt des Beitrags wird ein für den Untersuchungsgegenstand erst zu modellierendes ,Populäres ${ }^{6}$ sein. Zum

gilt, soll ihm damit nicht abgesprochen werden; anders als bei Ralf Georg Bogner, Der Autor im Nachruf. Formen und Funktionen der literarischen Memorialkultur von der Reformation bis zum Vormärz, Tübingen, Niemeyer, 2006, S. 25 werden Nachrufe hier aber nicht als literarische Texte verstanden.

3. - Vgl. R. G. Bogner, Der Autor im Nachruf, a.a.O., S. 10.

4. - Vgl. Thomas Goetz, Poetik des Nachrufs. Zur Kultur der Nekrologie und zur Nachrufszene auf dem Theater, Wien/ Köln/ Weimar, Böhlau, 2008, S. 29-54.

5. - Nach wie vor maßgeblich sind die Arbeiten von Stefan Brunn, AbschiedsJournalismus. Die Nachrufkultur der Massenmedien, Münster, LIT, 1999; Ralf Georg Bogner, „Der Nachruf als literarische Gattung. Möglichkeiten und Grenzen einer Definition", in: Franz Simmler (Hrsg.), Textsorten deutscher Prosa vom 12.13. bis 18. Jahrhundert, Frankfurt a.M., Peter Lang, 2002, S. 39-51; R. G. Bogner, Der Autor im Nachruf, a.a.O.; T. Goetz, Poetik des Nachrufs, a.a.O. Die Beiträge von Anna Hanus - „,Kritischer Nachruf' - eine neue Textsortenvariante?“, Colloquia Germanica Stetinensia, 25 (2016), S. 265-283; dies., „Der Nachruf in der polnischen und der deutschen Presse anhand der Texte um den Tod von Marcel Reich-Ranicki“, in: Dorota Kaczmarek (Hrsg.), Politik-Medien-Sprache. Deutsche und polnische Realien aus linguistischer Sicht, Łódź, Universitätsverlag, 2016, S. 119-134 - arbeiten ihre Fragestellungen nach Textsortenvarietäten bzw. nach kulturspezifischen Unterschieden zwischen deutschen und polnischen Nachrufen auch $(2016$, a) bzw. vor allem $(2016, \mathrm{~b})$ an Nachrufen auf Reich-Ranicki ab.

6. - Verlässliche Informationen zu einem komplexen Phänomen finden sich bei Thomas Kühn, Robert Troschitz (Hrsg.), Populärkultur. Perspektiven und Analysen, Bielefeld, transcript, 2017; John Storey, „Was ist Populärkultur?“, in: Ebd., S. 41-62; Thomas Hecken/ Marcus S. Kleiner (Hrsg.), Handbuch Popkultur, Stuttgart, 
einen wird es über die Textsorte ,Nachruf', die seit dem 19. Jahrhundert als ,populäre Textsorte“ gilt 7 , sowie über die Medien ihrer Publikation und Distribution fassbar. Dass Nachrufe zu den meistgelesenen Texten in Tages- und Wochenzeitungen des Print- und online-Sektors gehören, ist nicht nur aufgrund des Bedürfnisses nach aktueller Information über das Ereignis des Todes / den Toten und einer qua dieser zu erlangenden sozialen Integration erklärbar, sondern auch aufgrund ihres affektiven Potenzials und der psychologischen Funktionalisierung vom ,Tod des anderen', der - obwohl oder gerade weil er auch der eigene hätte sein können - die Möglichkeit zur erleichternden Distanzgewinnung mit sich führt. Zum anderen und für diesen Beitrag interessanter, ist ,das ' Populäre Reich-Ranicki und seiner Literaturkritik zugeordnet. Während er selbst eine bewusst für sich beanspruchte Popularität positiv kommentiert z.B. in dem viel und nicht zuletzt in Nachruftexten zitierten Satz „Ich hatte Freude daran, dass jeder Tankwart mich kannte" 8 -, erscheint sie in Fremdzuschreibungen durchaus ambivalent: Reich-Ranicki firmiert aufgrund seiner Popularität einerseits als Repräsentant der deutschen Kulturnation und als großer Lehrer, andererseits als Entertainer und als Mann des Boulevards.

Wenn das Populäre eine konstante Kollokation Reich-Ranickis und seiner Literaturkritik ist, wie es der vorliegende Band veranschlagt, dann muss es auch in Nachrufen nachweisbar sein. Es ist daher zu fragen, wem oder was Popularität zugeschrieben wird, über welche textuellen und intertextuellen Verfahren sie hergestellt wird und welchen Wertungen sie unterzogen wird. Besondere Aufmerksamkeit kommt der mehrfachen zeitlichen Gerichtetheit des Nachrufs zu: Zu einem bzw. auf einen bestimmten Zeitpunkt, nämlich dem/den des Todes, verfasst, stellt er das Resümee eines Lebens / eines Werkes und die Zusammenfassung und Kommentierung alles dessen, was über dieses bereits gesagt wurde, dar. Darüber hinaus formuliert und steuert der Nachruf als gleichsam ,ultimative' Zuerkennung das, was künftig erinnert werden soll. Die NachrufTexte auf Reich-Ranicki sind daher nicht nur auf die Strategien ihrer Re- oder auch Gegen-Konstruktion bzw. -Inszenierung des Populären zu untersuchen, sondern rücken als Instrument der Popularisierung auch selbst in den Fokus. Darüber hinaus sind sie nach Funktionalisierungen zu befragen, die nicht in einem unmittelbaren situativen und pragmatischen Zusammenhang mit Reich-Ranickis Tod und seiner Memorialisierung stehen - etwa einer Verhandlung aktueller Fragen des Literaturbetriebs

Metzler, 2017; Olivier Agard/ Christian Helmreich/ Hélène Vinckel-Roisin (Hrsg.), Das Populäre. Untersuchungen zu Interaktionen und Differenzierungsstrategien in Literatur, Kultur und Sprache, Göttingen, V\&R Unipress, 2011.

7. - Vgl. T. Goetz, Poetik des Nachrufs, a.a.O., S. 16.

8. - Marcel Reich-Ranicki im Interview mit Iris Radisch, Die Zeit, 27.05.2010. 
wie jene nach dem Verhältnis von Literaturwissenschaft, -kritik und -journalismus zu einem Populären.

\section{Exkurs: Neunundfünfzig Zeichen. Reich-Ranickis Todesanzeige auf Twitter}

Reich-Ranickis Tod wird zunächst nicht in einer Tageszeitung, sondern - und dies ist, will man Bogner Glauben schenken, für die aktuelle Praxis der Memorialkultur nicht ungewöhnlich ${ }^{9}$ - im Internet bekannt gemacht. Am 18. September 2013 um 16:01 wird via Twitter der Satz gepostet: „Marcel Reich-Ranicki ist im Alter von 93 Jahren gestorben"10. Die Todesanzeige verzichtet auf positive Attribuierungen, Euphemismen oder Floskeln, der knappe Aussagesatz bietet keinen Raum für Affekte, die Individualität des Verfassers verschwindet hinter seiner Funktion als Inserent. Alles in allem werden die Konventionen der Textsorte ,Todesanzeige ${ }^{11}$ durch jene des Media- bzw. Serverdesigns (für Twitter insbesondere Kürze und Dialogizität) dominiert. $986 \mathrm{Mal}$ wird die von Frank Schirrmacher/@fr_schirrmacher erstellte und verbreitete Nachricht auf dem Mikroblog von Rezipienten retweetet, also als Kopie weitergegeben, mit den jeweiligen followers geteilt, die Information dadurch diffundiert. Unmittelbar darauf und nahezu im Minutenabstand folgen die ersten tweets: Sie stammen nicht nur und nicht primär von Privatpersonen, sondern von Vertretern der Nachrichtenagenturen, Medien oder von Politikern. Sie reichen von individuellen wie standardisierten (häufig „RIP“) Betroffenheitsgesten bis hin zur Zurückweisung und fordern - wohl nicht nur mit Blick auf juristische und gesellschaftliche Sanktionen, sondern auch aufgrund der Sensibilität des Nachrichtengenres und seines aktuellen, Gegenstandes ${ }^{6}$ (des prominenten Verstorbenen) - Verifizierung. Um 16:03, das heißt nur zwei Minuten nach der Todesnachricht, erfolgt eine ins Kollektive gewendete Trauernachricht von Frank Schirrmacher: „Wir trauern alle. Noch vor 2 Stunden habe ich ihn besucht"12. Konstituiert diese Meldung einerseits eine Trauergemeinschaft, indem sie über die Rekonstruktion

9. - Vgl. R. G. Bogner, Der Autor im Nachruf, a.a.O., S. 5.

10. — https://twitter.com/fr_schirrmacher/status/380330844914593794 (Zugriff am 30.05.2019).

11. - Vgl. Kathrin von der Lage-Müller, Text und Tod. Eine handlungstheoretisch orientierte Textsortenbeschreibung am Beispiel der Todesanzeige in der deutschsprachigen Schweiz, Tübingen, Niemeyer 1995 sowie Eva-Martha Eckkrammer: Die Todesanzeige als Spiegel kultureller Konventionen. Eine kontrastive Analyse deutscher, englischer, französischer, spanischer, italienischer und portugiesischer Todesanzeigen, Bonn, Romanistischer Verlag, 1996, auf die sich sämtliche Folgestudien beziehen.

12. — https://twitter.com/fr_schirrmacher/status/380330844914593794 (Zugriff am 30.05.2019). 
der Popularität des Verstorbenen Identität sichert, so beansprucht sie andererseits Exklusivität durch Intimität. Der Kritiker, dessen Sterben bereits in fiktionalen Texten vorweggenommen wurde, ${ }^{13}$ - ist nun tatsächlich und endgültig tot.

\section{Dreiundvierzig Texte. Reich-Ranicki in Nachrufen deutschsprachiger Tages- und Wochenzeitungen}

Eine repräsentative Zusammenstellung an Nachruf-Texten auf ReichRanicki findet sich in der Sonderausgabe des Pressespiegels Medien ${ }^{14}$. Die insgesamt achtzig dort verzeichneten Texte zum Tod von Reich-Ranicki wurden in einem Zeitraum von zehn Tagen publiziert: der erste noch am Tag seines Ablebens (18.09.2013) ${ }^{15}$, die letzten am 27.09.2013, dem auf die offizielle Trauerfeier folgenden Tag. Größtenteils handelt es sich dabei um Nachruftexte in der Form von Einzel-Nachruf und Nachruf-Spiegel (also Zusammenstellungen von Auszügen aus bestehenden Nachruftexten), aber auch um Interviews, Reportagen, Wiederabdrucke aus ReichRanickis Publikationen, Sendungshinweise etc. Abgesehen von einzelnen Agentur-Meldungen stammen sie zum größeren Teil aus gedruckten, zum kleineren Teil aus online verfügbaren Zeitungen: aus Tages-, Sonntags- und Wochenzeitungen, aus regionalen und überregionalen Zeitungen, aus mit Blick auf Reich-Ranickis Staatszugehörigkeit und Lebensraum in- und ausländischen Zeitungen (Schweiz, Österreich, England, Frankreich, USA), aus Qualitäts- und sehr wenigen Boulevardzeitungen. Darüber hinaus zeigt sich eine durch die Biografie des Verstorbenen indizierte regionale Konzentration der Berichterstattung im Raum Frankfurt am Main. Mit der Prominenz und Popularität des Verstorbenen korrelieren neben dem Faktor ,Verbreitung' die Gesamtanzahl der Nachrufe, ihre Zahl und Frequenz im einzelnen Organ ${ }^{16}$, ihr Umfang ${ }^{17}$ sowie ihre Positionierung in der Zeitung:

13. - Beispielsweise in Martin Walsers Roman Tod eines Kritikers (2002), in dem Ehrl-Königs ,Tod“ widerrufen wird, oder, für den aktuellen Beitrag interessanter, in Heißenbüttels Nachruf bei Lebzeiten (1988), der den Tod Reich-Ranickis probeweise und aus der subjektiven Perspektive des Gegners herstellt. Vgl. Helmut Heißenbüttel, „Nachruf bei Lebzeiten“, Text und Kritik, 100 (1988), S. 26-28, hier S. 28: „Er ist für mich ein Gestorbener."

14. - Vom 19. und 20.09.2013, erstellt von Klaus Makoschey, sowie vom 23. und 27.09.2013, erstellt von Christa Matthäi.

15. - Inwieweit die kurz nach Reich-Ranickis Tod veröffentlichten Nachrufe bereits vorrätig waren (eine im Feuilleton gängige Praxis, vgl. St. Brunn, AbschiedsJournalismus, a.a.O., S. 105-116), kann nicht verifiziert werden.

16. - Die mit Abstand meisten, nämlich zwölf Texte wurden in der FAZ / FAZ Sonntag abgedruckt, was sich aus der langjährigen personellen Verbundenheit mit Reich-Ranicki erklären lässt, gefolgt von der Süddeutschen Zeitung mit sieben und der Frankfurter Rundschau mit sechs Beiträgen.

17. - Der mit 4877 Wörtern, das sind grob gerechnet 35000 Zeichen, längste 
entweder auf der Titelseite / im Mantelteil, auf der dritten Seite oder - sehr häufig - im Feuilleton bzw. im Kultur- oder Literaturteil, seltener in den Rubriken Wissen \& Bildung sowie Leute. Die Nachrufe auf Reich-Ranicki sind Chefsache: Sie stammen zum größten Teil von festen Mitarbeitern der jeweiligen Zeitungen - von Redakteuren, Ressortleitern und Kritikerkollegen, häufig sind diese Reich-Ranicki auch freundschaftlich verbunden -, kaum aber von Autoren literarischer Texte ${ }^{18}$. Die Nachrufer sind, wo nicht von der Redaktion gestellt oder beauftragt, jedenfalls von dieser sanktioniert.

Für die folgenden Überlegungen wurde ein Korpus von 43 Texten erstellt, das für die Nachrufe des Zeitraums vom 18. bis zum 27.09.2013 als repräsentativ gelten kann. Es umfasst sowohl Texte, die primär (und hier wiederum zum allergrößten Teil ausschließlich) für die Publikation in Tages- bzw. Wochenzeitungen geschrieben wurden, als auch Reden, die im Rahmen der rituellen Begräbnisfeierlichkeiten öffentlich gehalten und sekundär in Zeitungen abgedruckt wurden. Diesen kommt insofern eine Sonderstellung zu, als sie für eine konkrete zeremonielle Handlung konzipiert und in deren unmittelbarem situativen Kontext verortet sind: dem offiziellen Trauerakt, der am 26.09.2013 um $15 \mathrm{Uhr}$ in der Trauerhalle des Frankfurter Hauptfriedhofs in der physischen Präsenz des Verstorben und zahlreicher Trauergäste abgehalten wird. Konstitutiver Bestandteil der weltlichen theatralen Feier, deren Dramaturgie in einem seinerseits veröffentlichten Programm ${ }^{19}$ niedergelegt ist, sind - neben zeichenhaft-symbolischen Artikulationen der Trauer durch Requisiten (Blumen, Kerzen...) und musikalische Darbietungen (Werke von Bach, Schumann, Puccini) - die insgesamt acht Nachruf-Reden ${ }^{20}$. Die Flüchtigkeit des gesprochenen Wortes, die Ereignishaftigkeit des Moments wird in Schrift und Bild transformiert ${ }^{21}$, über Speichermedien konserviert und, für Trauerreden unüblich ${ }^{22}$ und wohl durch die Prominenz des Verstorbenen motiviert, über die Tagesund Wochenpresse auch für eine große Öffentlichkeit verfügbar gemacht. Als Nachruf-Texte stehen sie auch im Blick der folgenden Untersuchung. Nicht (mehr) berücksichtigt werden dagegen Retrospektiven auf die öffentliche Trauerfeier, da sie nicht primär eine Reaktion auf den Tod

erschien am 21.09.2013 im Spiegel.

18. - Als eine Ausnahme ist zu nennen: Martin Walser, „Ich bin ihm nahe“, Die Zeit, 26.09.2013, https://www.zeit.de/2013/40/nachruf-reich-ranicki-walser (Zugriff am 30.05.2019). Der Text findet sich nicht in der Presseschau.

19. - https://www.maths.ed.ac.uk/ v1ranick/surgery/programme.pdf (Zugriff am 30.05.2019).

20. - Neben Frank Schirrmacher, Rachel Salamander, Salomon Korn, Thomas Gottschalk, auf die dieser Beitrag fokussiert, Vertreter aus Stadt- und Landespolitik.

21. - Eine gut einstündige Audiodatei der Zeremonie ist verfügbar unter: https:// www.maths.ed.ac.uk/ v1ranick/funeralaudio.mp3 (Zugriff am 30.05.2019).

22. — Vgl. T. Goetz, Poetik des Nachrufs, a.a.O., S. 16. 
eines Menschen, sondern - gleichsam als Reaktion 2. Ordnung - eine Reaktion auf eine Reaktion auf den Tod eines Menschen darstellen.

\section{SAGEN und MACHEN. Textlinguistische und -pragmatische Überlegungen des Nachrufens am Beispiel von Reich-Ranicki}

Für die Beantwortung der Frage, was der Nachruf wem auf welche Art und Weise kommuniziert, ist (s)eine mehrfache Prädikation von Interesse. Indem der Nachrufer über den Verstorbenen spricht/ schreibt, spricht/schreibt er auch über sich und über die Gruppe der Trauernden, in die er sich mit seinem Text performativ stellt, in der er sich aber auch gleichzeitig exponiert: Mit der Aufgabe, sich öffentlich zum Tod Reich-Ranickis zu äußern, hat er die gesellschaftlich relevante Rolle des legitimierten Sprechers bzw. Schreibers übernommen, der sich gleichzeitig selbst inszeniert - vorneweg als ,guten', d.h. den Konventionen der Textsorte folgenden und die Erwartungen der Gesellschaft erfüllenden Nachrufer. Anders als die Todesanzeige ist der Nachruf weniger durch strukturelle Musterhaftigkeit geprägt als durch inhaltlich und funktional bestimmte Komponenten, die in Abfolge, Gewichtung und elokutionärer Gestaltung stark variieren können. Von den bei Goetz genannten ${ }^{23}$ textsortenkonstitutiven Teilen sind lamentatio, consolatio sowie gratiarum actio und damit die affektstärksten Momente in den Nachrufen auf Reich-Ranicki nur wenig ausgeprägt bzw. fehlen ganz. Die Familie, deren persönlicher Verlust und deren Trauer rücken nur selten in den Fokus, und auch explizite und direkte Artikulationen von (eigener) Trauer oder von Trost, der über den Effekt individueller Figurenpräsenz im Text ${ }^{24}$ durch Nennung, Ansprache oder Prosopopöie ${ }^{25}$ hinausgeht, bleiben die Ausnahme. Nur wenige Nachrufe auf Reich-Ranicki adressieren den Verstorbenen im Du. Die meisten sprechen über ihn als Abwesenden, dessen Fehlen für die Gegenwart festgestellt und, unter Betonung der Endgültigkeit des Todes, für die Zukunft prognostiziert wird ${ }^{26}$. Gleichzeitig formulieren die Nachrufe, selbst Form und Manifestation des Gedenkens ${ }^{27}$, auch Strategien gegen

23. - Vgl. ebd., S. 25.

24. - Vgl. ebd., S. 92. Intensiviert wird die Präsenz durch die Nennung von Eigenschaften und Verhaltensweisen, die als ,typisch ${ }^{\star}$ markiert sind: die Frage nach Neuem, das rollende R / das gelispelte S, die Anrede „mein/e Liebe/r“, das Urteil „grässlich“" etc.

25. - Darunter auch vorgestellte Reaktionen des Verstorbenen auf den jeweiligen Nachruf.

26. - Die Möglichkeiten von Ersetzbarkeit und Nachfolge werden toposhaft zurückgewiesen.

27. - T. Goetz, Poetik des Nachrufs, a.a.O., S. 30. 
das Vergessen: Sie versprechen ein immerwährendes Andenken, das in der Einzigartigkeit des Verstorbenen (mit oder ohne Nennung seiner Alleinstellungsmerkmale) begründet ist. Seinen Ausdruck findet es sowohl in individuellen Erinnerungen als auch in organisierten und inszenierten Formen des Gedenkens, insbesondere einer für Oktober 2013 angedachten und im Juni 2014 (einen Tag vor Reich-Ranickis Geburtstag) abgehaltenen offiziellen Gedenkfeier in der Frankfurter Paulskirche. Zum Träger einer „semiotische[n]“ und ,ontische[n] Kontinuität" 28 Reich-Ranickis über den Tod hinaus wird das ,Werk': die abstrakte Summe aus fast 100 Büchern, 650 Essays, unzähligen Rezensionen und nicht zuletzt aus medialen Auftritten.

Die zentralen Bestandteile der Nachrufe auf Reich-Ranicki sind vita und laudatio. Für den biografischen Abriss hat Reich-Ranicki mit seiner 566 Seiten langen, 1999 in der Deutschen Verlags-Anstalt Stuttgart erschienenen Autobiografie Mein Leben eine umfassende und durch die eigene Verfasserschaft maximal authentifizierte und autorisierte Vorlage bereitgestellt. Auf sie referieren, direkt oder indirekt, nahezu alle Nachrufe. Mit dem Leben der realen Person bzw. deren öffentlichem Image erzählt der Nachruf auch die von Reich-Ranicki selbst begonnene Narration (s)eines Lebens wieder und führt diese aus Anlass seines Sterbens und von diesem her zu Ende. Dennoch ist der Tod Reich-Ranickis selten der Anfangspunkt des Sprechens über ihn. Nur wenige Nachruf-Texte setzen damit ein, indem sie explizit darauf Bezug nehmen, ,exklusiv' Zeugenschaft über sein Sterben ablegen 29 oder dieses für den Verstorbenen beurteilen ${ }^{30}$; stattdessen dominieren unmittelbare Einstiege: episodische Alltagsgeschichten im Stil der Erlebnis- oder Erinnerungserzählung, Beschreibungen charakteristischer Situationen/Szenen, illustrierende Anekdoten sowie Bonmots des Literaturkritikers stehen neben Schilderungen historischer Ereignisse und politischer Konstellationen in ihrer Wahrnehmung durch und ihrer (durchaus schicksalhaften) Relevanz für Reich-Ranicki. Aus einer Zusammenschau der Nachruf-Anfänge lassen sich sowohl die wesentlichen inhaltlichen Momente der Biografie als auch Verfahren ihrer Darstellung in den Nachruftexten abstrahieren. Anders als in der Lebens-Erzählung Reich-Ranickis sind die Biographeme weder ,vollständig' noch entlang einer von der Geburt zum Tod verlaufenden Zeitlinie angeordnet. Als Kriterien ihrer Selektion fungieren ein für den Nachruf-,Gegenstand' (den Verstorbenen) als charakteristisch, ein im Hinblick auf das Darstellungsziel der Würdigung und

28. - Vgl. ebd., S. 44.

29. - Wie etwa die Sterbeszene in der BILD-Zeitung.

30. - Singulär ist die Einschätzung als Erlösung aus einer lebenslangen Traumatisierung. 
der Memorialisierung als relevant Erachtetes. In unterschiedlicher Schwerpunktsetzung und Detailgenauigkeit fokussieren die Texte einerseits auf Reich-Ranickis (späte) Karriere als Literaturkritiker, andererseits auf sein durch die Erfahrungen von Nationalsozialismus und Flucht geprägtes, jüngeres Erwachsenenalter. Als zentrale Momente rücken Reich-Ranickis Ausweisung aus Deutschland 1938, das Leben im Warschauer Ghetto, die Erstürmung des Judenrats durch SS-Männer und die Order zur Deportation der jüdischen Bewohner, die unmittelbar darauf folgende Hochzeit mit Teofila / „Tosia“ Langnas, die Flucht aus dem Ghetto und das Leben im Versteck bei Bolek und Genia Gawin in den Blick. Reich-Ranickis letzte Flucht aus dem kommunistischen Polen und seine Ankunft in Berlin am 21.07.1958 werden zum Wendepunkt der Handlung, zum Neueinsatz im doppelten cursus von Lebens- und Erfolgserzählung. Noch stärker als in der primär informierenden, charakterisierenden und kontextualisierenden vita rückt Reich-Ranicki in der laudatio über unterschiedliche Professionen und Funktionen in den Blick: als Literaturkenner, -stratege und -kritiker, als Literaturjournalist, -redakteur und -chef für Tages- und Wochenzeitungen (insbesondere die Zeit und die FAZ), als Autor seiner Biografie und Herausgeber der Frankfurter Anthologie, als Förderer junger Autoren, Juror und vielfacher Preisträger, als Lehrer und Erzieher, als Moderator und Diskutant, Schauspieler und Medienstar von ZDF (Das literarische Quartett), $A R D$ (Verfilmung von Mein Leben) und, nach der Zurückweisung des Fernsehpreises 2008, auch von You Tube, aber auch als öffentliche Stimme abseits des Literaturbetriebs, z.B. als Holocaust-Redner vor dem Deutschen Bundestag 2012. Vita und laudatio werden in den NachrufTexten auf Reich-Ranicki weder linear geführt noch manifestieren sie sich als kohärente und voneinander abgrenzbare Teile. Sie sollen daher für eine textlinguistisch und -pragmatisch orientierte Analyse gemeinsam betrachtet werden.

Konstitutiv für die Nachrufe auf Reich-Ranicki ist die enge Korrelierung des Profils des späten mit den Erfahrungen des jungen Reich-Ranicki, seiner öffentlichen Leistung (als Literaturkritiker) mit seinem (privaten) Leben, als deren existenzieller ,Störfaktor' die ideologischen Verfehlungen im Europa der 1930er, 40er und 50er Jahre gelten müssen. Über die Fokussierung der Kritiker-Instanz und die problematische Kategorie der ,Persönlichkeit', für die sich die Frage der Zugänglichkeit nicht nur aufgrund der inszenatorischen Qualität der öffentlichen Kritiker,figur ${ }^{\circ} 31$ Reich-Ranickis stellt, lancieren sie nicht nur ein subjektivistisches Bild von Reich-Ranicki und seiner Literaturkritik, sondern sie reproduzieren damit auch eine gängige

31. - Analog zur Autorfigur verstanden als das öffentliche Konstrukt des Kritikers und wie diese den Unterschied zum -subjekt betonend. 
Praxis populärer Interpretation. Doch auch abseits von methodischen Vorbehalten gegen einen Biographismus positivistischer Prägung sowie eine Hermeneutik und Psychologisierung im Sinne Schleiermachers (bzw. deren Reduktionsstufen) bleibt die Argumentation nicht widerspruchsfrei. Wird einerseits Reich-Ranickis Doing Literaturkritik aus seinem Leben erklärt, verweisen die Nachrufe andererseits auf den ,anderen', den ,eigentlichen' (den geselligen, freundlichen, charmanten und zärtlichen) Menschen hinter dem (harten, bisweilen auch ungerechten) Kritiker. Reich-Ranicki wird in einer im Geschichts- und Erinnerungsdiskurs der letzten Jahrzehnte zwar kritisch reflektierten, ${ }^{32}$ in einem politisch-moralischen Sinn aber unangreifbar gewordenen Täter-Opfer-Dichotomie positioniert, die auch dann nicht aufgegeben wird, wenn die Lebensgeschichte mit dem ethischen Diskursgebot der Textsorte ,Nachruf' kollidiert wie am Beispiel von Reich-Ranickis (Spionage?)Tätigkeit für den kommunistischen Geheimdienst in England. Trotz ihrer Individualität und Exzeptionalität wird seine Biografie als für das 20. Jahrhundert ,charakteristisch' identifiziert und auch für die ,große' Geschichte, insbesondere die Geschichte Deutschlands, funktionalisiert. Indem sie am Beispiel Reich-Ranickis das aktuelle Verhältnis Deutschlands zu seiner nationalsozialistischen Vergangenheit skizzieren und die Bemühungen um Aufarbeitung dokumentieren, werden die Nachrufe zum Podium für eine generell als aktiv, kritisch und erfolgreich dargestellte deutsche Gedächtnisund Erinnerungskultur. Sie artikulieren das Paradoxon des von den Nationalsozialisten vertriebenen, verfolgten und mit der Ermordung bedrohten polnischen Juden, der Frankfurt zu seinem Lebenszentrum gewählt hat und, indem er ,den Deutschen' ihre ,eigene Literatur nähergebracht und erklärt hat, Karriere gemacht hat und zu einem der prominentesten Deutschen geworden ist. Die Stilisierung des in menschlicher Größe verzeihenden, in kultureller Zuwendung gebenden und an die Humanität mahnenden Reich-Ranicki bzw. des in Demut und Dankbarkeit annehmenden Deutschlands (u.a. im Bekenntnis kollektiver Schuld und in der Akzeptanz der Täterrolle) fällt deutlich aus. An keinen anderen Stellen zeigen die Nachruf-Texte ein vergleichbares Pathos, das mitunter die Grenze zur Ironie überschreitet.

Die Akzentuierung des Literaturkritikers Reich-Ranicki erfolgt zunächst über die Zuordnung zu einer Gruppe und durch die anschließende Herstellung von Distinktion zu den übrigen Gruppenmitgliedern, indem ihm eine herausragende Position in den diachronen und synchronen Arrangements von Epoche, Nation und Kultur/Kulturbetrieb zugeschrieben wird. Seine Überlegenheit wird in seinen intellektuellen

32. - Für eine differenzierte Sicht vgl. u.a. Aleida Assmann, Der lange Schatten der Vergangenheit. Erinnerungskultur und Geschichtspolitik, München, Beck, 2006, S. 72-84. 
(Artistik, Klugheit, Geistesgegenwart, Witz, Esprit), dialektischen (Streitbarkeit) und rhetorischen (Redegewalt, Scharfzüngigkeit) Fähigkeiten, in einer ihm eigenen Verve (Energie, Enthusiasmus, Leidenschaft, Besessenheit), in seinen Strategien von Darstellung (Szene, Spektakel, Schauspielerei, Unterhaltung) und Vermittlung (Publikumsorientierung, Aufbereitung), vor allem aber in seiner Macht und seinem Einfluss begründet. Als Instrumente ihrer Herstellung fungieren, neben den bereits in anderem Kontext genannten narrativen Formaten, auctoritas (also Aussagen kompetenter und/oder kanonisierter Mitglieder unterschiedlicher Gruppen wie Literaturkritiker, Autoren, aber auch Politiker) über den Toten, vielfältige Attribuierungen und epitheta ornantia, Personifikationen, Metaphorisierungen, Hyperbeln und Vergleiche Reich-Ranickis mit anderen verdienten Instanzen oder Persönlichkeiten, in deren Reihe er zu stellen ist - etwa in die Tradition der (,jüdischen') Literaturkritik bzw. deren maßgeblicher Vertreter wie Alfred Kerr, Alfred Polgar, Siegfried Jacobsohn, Kurt Tucholsky - oder die er in der Universalität und Absolutheit des Superlativs als größter, geachtetster etc. Literaturkritiker auch überbietet. Von den Affekten steht, der Textsorte ,Nachruf' und seiner sprichwörtlich gewordenen Maxime „de mortuis nil nisi bene" 33 wie der Texthandlung des Lobens gleichermaßen entsprechend, offene Bewunderung im Vordergrund. Für die komplementäre Kritik bleibt dementsprechend wenig Raum: Explizit benannt und kritisch reflektiert werden Negativa nur selten und bestenfalls punktuell ${ }^{34}$. Stattdessen werden Aspekte mit negativer Valenz häufig ausgespart, in reduzierter, objektivierter Form in die Nachrufe aufund damit hingenommen, in ihrer Qualität und Quantität abgeschwächt, als direkte Folgen historisch-situativer Einwirkungen dargestellt ${ }^{35}$, plausibilisiert und dadurch - wo nicht ins Positive gewendet, so doch ,verstehbar' gemacht -, oder sie werden durch ein gleichzeitig vorhandenes Positives aufgewogen und dadurch quasi kompensiert.

\section{NACHGESAGTE Popularität. Zu einer ambivalenten Kategorie in Reich-Ranickis Nachrufen}

Die Nachrufe referieren auf einen sichtbaren, von der Öffentlichkeit mit der Gratifikation ,Beachtung' versehenen, in seiner Bekanntheit bekannten ${ }^{36}$ und darüber wiedererkennbaren Reich-Ranicki und

33. - Mittlerweile zugunsten von kritischen Momenten im Nachruf relativiert, vgl. u.a. T. Goetz, Poetik des Nachrufs, a.a.O., S. 26.

34. - Z.B. an Reich-Ranickis Geheimdiensttätigkeit (Vita) und an den literaturkritischen Fehden.

35. - Z.B. Reich-Ranickis Rastlosigkeit und Angst, seine Sehnsucht nach Akzeptanz und Anerkennung, sein Durchsetzungs- und Darstellungsehrgeiz, aber auch seine Härte.

36. - Vgl. Thomas Schierl (Hrsg.), Prominenz in den Medien. Zur Genese und 
konsolidieren damit eine zu Lebzeiten vom Publikum zuerkannte, über die Grenzen von Nation, Berufs- und Interessensgruppen hinausgehende Prominenz. Als eines von verschieden codierten und zirkulierten Formaten positionieren sich Nachrufe in einem Diskurs der Prominenz, auf den sie sich beziehen, den sie aber auch mitgestalten. Wie das Interview, der (Gast)Kommentar, die Talkshow und Formen des Life-Writings setzen sie Relevanz voraus und können damit auch als Textsorten der Prominenz gelten. Von besonderem Interesse für die aktuelle Fragestellung ist die Wechselwirkung von Prominenz mit einem zweiten Phänomen: einer ebenfalls über eine (mediale) Aufmerksamkeits-Öffentlichkeit bzw. als deren Effekt zu denkende Popularität. Auf der Bezeichnungsebene werden ,Prominenz ${ }^{637}$ und ein zusätzlich an die Kategorie der ,Beliebtheit' gebundenes ,Populäres' für Reich-Ranicki in der Formulierung vom „Popstar der Literaturkritik“38 zusammengeführt. Die auch aus anderen Bereichen, insbesondere der Wissenschaft, bekannte Phrasierung konstruiert den Kritiker als von anderen abgehobene Person bzw. deren Image ${ }^{39}$; gleichzeitig stellt sie in der metaphorischen Verbindung von populärkulturellem Phänomen und ,solider' Literaturkritik zwei unterschiedliche Domänen in ihrer Gegensätzlichkeit aus.

Popularität dürfte nicht nur eine der häufigsten Kollokationen Reich-Ranickis sein, sondern auch jene mit der größten Ambivalenz; beides wird durch Nachruf-Texte gespiegelt. Die Nachrufe entwickeln zunächst ein stark quantitativ geprägtes und insofern dem Prominenten nahestehendes Verständnis des Populären als das, was viele erreicht. Systemisches Potenzial erhält ein derart bestimmtes Populäres dadurch, dass es über Berufs- und Interessensgemeinschaften hinaus Rezipienten akquiriert und zu neuen, ,funktionsspezifische[n] Klientenrollen“40, hier: zu Lesern/Zuschauern von Reich-Ranickis Literaturkritik, im Idealfall auch zu Lesern von dort besprochenen Büchern oder, allgemeiner, von ,Literatur' formiert. Nachrufe auf Reich-Ranicki sprechen von einem nicht notwendigerweise bzw. charakteristischer-

Verwertung von Prominenten in Sport, Wirtschaft und Kultur, Köln, van Halem, 2007, S. 16.

37. - Deren besonders herausragende Vertreter und ihre performativen Rollen werden zur Gruppe der ,Stars` zusammengefasst, vgl. Julia Wippersberg, Prominenz. Entstehung. Erklärungen. Erwartungen, Konstanz, UVK, 2007, S. 282.

38. - P. Schneider, „Marcel Reich-Ranicki wird 80“, a.a.O. Die Benennung „Popstar" ist auch titelgebend für das 9. Kapitel in der bislang umfangreichsten Biografie: Uwe Wittstock, Marcel Reich-Ranicki, Die Biografie, München, Hanser, 2015, S. 5.

39. - Vgl. J. Wippersberg, Prominenz, a.a.O., S. 35.

40. — Urs Stäheli, „Bestimmungen des Populären“, in: Kirsten Huck, Karsten Zorn (Hrsg.), Das Populäre der Gesellschaft. Systemtheorie und Populärkultur, Wiesbaden, Verlag für Sozialwissenschaften, S. 306-321, hier: S. 309. 
weise nicht gegenstandsaffinen Massenpublikum und einer breiten Öffentlichkeit an Rezipienten. Sie nennen (wenn auch recht unterschiedliche) Reichweitenzahlen für seine Zeitungspublikate, Auflagenund Verkaufszahlen für seine Autobiographie sowie Einschaltquoten für seine Fernsehauftritte, insbesondere im Literarischen Quartett ${ }^{41}$, und forcieren damit Reich-Ranickis Rolle als Verbreiter von Literatur. Eine besondere Relevanz für seine Popularität wird den Massenmedien zugeschrieben: dem Fernsehen, seinem Verhältnis zu und seiner (als sein Popularisator und Beschädiger) sehr unterschiedlich beurteilten Auswirkung auf Reich-Ranicki, aber auch Tages- und Wochenzeitungen (insbesondere der Zeit, der Welt und der FAZ), die als Begründer von Reich-Ranickis früher (ab den 1960er Jahren), vielleicht auch ,eigentlicher' Popularität bewusst von diesem abgesetzt werden und über die eine , andere', ,wertigere' (?) Qualität von Popularität hergestellt wird.

Für eine qualitative Bestimmung (s)einer Popularität fokussieren die Nachrufe vor allem auf Reich-Ranicki als Kritiker und Vermittler von Literatur. Sein Name wird zum „Markenzeichen“42 für eine Literaturkritik, der über ihre massenmediale Distribution und ihren massenhaften Konsum ökonomische Aspekte auch über einen materiellen Profit hinaus zu kommen, wie sie Pierre Bourdieu in seinem Theorem vom sozialen Kapital modelliert ${ }^{43}$. Reich-Ranickis Popularität wird zum Marktplatz von Verlagen und Buchhandel, zur Plattform von Ausgaben und Auflagen; sie ermöglicht aber auch Gruppenzugehörigkeit durch die Teilhabe an und den Austausch über etwas (hier: Literatur und ihre Kritik, aber auch Reich-Ranicki als deren Repräsentant) sowie - eine wie auch immer konturierte - ,Bildung' und die durch diese erworbene Anerkennung. Dass Reich-Ranickis literaturkritische und -vermittlerische Praxis in Nachrufen dennoch ambivalent bewertet wird, hat mehrfache Ursachen. Zunächst ist die Wahrnehmung stark auf seine Person zentriert: auf sein wirksames Äußeres, auf seine Emotionalität und sein Charisma, auf seine rhetorischen Fähigkeiten und seine darstellerischen Qualitäten als Schauspieler auf der Bühne der Literaturkritik, kurz: auf seine Praktiken der Theatralisierung und Inszenierung und den daraus resultierenden Unterhaltungswert seiner Performances. Mit der Attraktion der Person übernehmen Literaturkritik und -vermittlung auch deren Hypothek: Reich-Ranickis literaturkritisches (insbesondere das der literarischen [Ab]Wertung) und -vermittlerisches Handeln wird ebenso widersprüchlich beurteilt

41. - Zahlen finden sich bei U. Wittstock, Marcel Reich-Ranicki, a.a.O., S. $286 f$.

42. - Ebd., S. 155.

43. - Vgl. Pierre Bourdíeu, „Ökonomisches Kapital - Kulturelles Kapital Soziales Kapital“, in: Ders., Die verborgenen Mechanismen der Macht. Schriften zu Politik \& Kultur 1, Hamburg, VSA, 2015, S. 49-80. 
wie der Akteur selbst. Nachrufe konstatieren ein kalkuliertes writing to the audience, eine Anbiederung an die Öffentlichkeit sowie eine (unheilige) ${ }^{44}$ Allianz des Kritikers mit seinem Publikum. Die beiden wichtigsten Kritikpunkte betreffen die Methode von Reich-Ranickis Literaturkritik, bzw. richtiger: deren Fehlen, sowie ihre (macht-)diskursiven Implikationen. In Nachrufen ist die Rede von Emotionalität und Radikalität, von Unsachlichkeit und Schematisierung, aber auch von Absolutismus und Dogmatismus. Immer wieder thematisieren und reflektieren die Nachrufe Reich-Ranickis eindeutige und dichotomischen Entwürfen verpflichtete Zuordnungen, die nicht zur Disposition stellen, sondern die schon (auch für den Leser) entschieden haben, seine apodiktischen (und dabei subjektiven) Urteile, die den Rezipienten spezifische vorgefertigte Lesepositionen präsentieren, die von diesen übernommen werden sollen. An der hegemonialen Dimension von Reich-Ranickis literaturkritischer Praxis vermag auch ein dem Lese-, vor allem aber auch ein dem Nicht-, Noch-nicht- oder Gerade-mal-eben-Lesepublikum, jedenfalls aber dem Publikum der Kritik unterstelltes ,Bedürfnis' nach Orientierung durch Selektion und Sortierung nichts zu ändern: Reich-Ranickis Popularisierungspraktik erscheint in Nachrufen auch als bewusst (und für den eigenen Vorteil) genutzte Taktik.

Paradoxerweise liegt, und das lässt sich auch an Reich-Ranickis Literaturkritik beobachten, die Ursache für die ambivalente Beurteilung des Populären in seiner größten Leistung: in seiner Orientierung am Publikum. Indem es Nicht-Publika zu Publika macht, ermöglicht das Populäre einerseits sehr vielen Zugang zu Literaturkritik und qua dieser vielleicht auch zu Literatur; andererseits bedeutet Inklusion für etablierte Publika den Verlust an Exklusivität und an Möglichkeiten zur Distinktion. Dementsprechend findet Reich-Ranicki seine größten Kritiker ${ }^{45}$ in den Vertretern der Literaturwissenschaft und einer theoretisch fundierten Literaturkritik, denen seine Kritik aufgrund ihres methodenfernen Konzepts und ihrer aus traditioneller Sicht gegenstands- und professionsinadäquaten Praktiken der Vermittlung (z.B. im Literarischen Quartett $)^{46}$ zum Gegenentwurf wird ${ }^{47}$. Mit den Differenzen sind auch

44. - Die Frage, gegen wen oder was sie gerichtet ist, bleibt offen.

45. - Abgesehen von den kritisierten und widerkritisierenden Autoren, die hier als direkt ,Betroffene' nicht diskutiert werden sollen.

46. - Vgl. Marcus S. Kleiner, „Philosophie im Fernsehen / Philosophie des Fernsehens. Metamorphosen philosophischen Wissens im Fernsehformat Precht", in: Marcus S. Kleiner, Thomas Wilke (Hrsg.), Populäre Wissenschaftskulissen. Über Wissenschaftsformate in Populären Medienkulturen, Bielefeld, transcript, 2017, S. 99-128.

47. - Ausgelotet wurde diese Problematik am Beispiel von Wissenschaft und Popularität / von populärem Wissen u.a. von Carsten Kretschmann (Hrsg.), Wissenspopularisierung. Konzepte der Wissensverbreitung im Wandel. Berlin, 
die Hierarchien festgelegt: Wenngleich die Nachrufe das (insbesondere vermittlerische) Potenzial von Reich-Ranickis populärer Literaturkritik einerseits erkennen und auch würdigen können, verfestigen sie andererseits hegemoniale Strategien der Wertung, indem sie seine Literaturkritik - trotz ihres zum allergrößten Teil hochkulturellen Gegenstandes aufgrund eines verminderten Anforderungsgrades der populären Kultur zuschlagen und damit über das Verfahren des Ausschlusses als das ,Andere“ der Hochkultur definieren. Ebenso wenig wie Reich-Ranicki die Kategorien von Hoch- und Populärkultur für sich theoretisch reflektiert hat (seine Positionierung ist nicht nur aus der Zusammensetzung seines vierundfünfzig Bände umfassenden Kanons ${ }^{48}$ ablesbar, sondern bereits aus dem Ansinnen, einen solchen zu erstellen), findet Skepsis gegenüber einer überkommenen Unterscheidung, wie sie die Postmoderne entwickelt hat, in den Nachrufen auf Reich-Ranicki Raum.

\section{NACHRUFEN und VOR(HER)SAGEN. Ein Fazit}

Die Untersuchung eines repräsentativen Textkorpus ergibt für Nachrufe auf Reich-Ranicki in ausgewählten Tages- und Wochenzeitungen folgendes Profil: Nachrufe zeigen sich - abgesehen von Varianzen in Umfang, Anordnung und Elokution - in ihren textlinguistischen (inhaltliche Akzentuierung von Leben und Werk, geringe Stimulierung bzw. Expression anlassspezifischer Affekte wie Trauer und Trost) und -pragmatischen (Lob und Würdigung, Sicherung von Identität, Konstitution von Erinnerung) Merkmalen weitgehend konventionell, in der engen Korrelation von Leben und Werk, die biografistische, hermeneutische und psychologisierende Züge trägt, traditionell-restaurativ. In der Charakteristik Reich-Ranickis und seiner Literaturkritik dominieren jene Merkmale, die ihm bzw. ihr von verschiedenen Akteuren des Literaturbetriebs bereits zu Lebzeiten zugeschrieben wurden, darunter auch die Publikums-Phänomene von Prominenz und Popularität. Das Populäre wird von Nachrufen als das, was vielen bekannt ist und was viele mögen, als - durchaus auch durch ökonomische Aspekte bestimmte - Massenkultur und als das Andere einer so genannten Hochkultur entworfen. Popularität in dem einen oder anderen Sinn ist nicht nur die häufigste Zuschreibung an Reich-Ranicki, sondern auch diejenige, die am meisten polarisiert. Die Bewertungen von Reich-Ranickis Popularität stehen in der Tradition von akademischer Literaturwissenschaft und theoriegeleiteter Literaturkritik bzw.

Akademie, 2003; Arne Schirrmacher, „Nach der Popularisierung. Zur Relation von Wissenschaft und Öffentlichkeit im 20. Jahrhundert", Geschichte und Gesellschaft 34 (2008), S. 73-95.

48. - Als Kriterium der Auswahl gilt neben literarischer Qualität auch Lesbarkeit. 
von Erziehung und Vermittlung, deren (hauptsächlich) negative bzw. positive Sichtweise sie - sei es, um sie zu affirmieren, sei es, um sie zu widerlegen - reproduzieren. Zumeist zeigen Nachrufe entweder die eine oder die andere Sichtweise des Populären, selten werden beide innerhalb eines Textes thematisiert, miteinander konfrontiert oder reflektiert. Wenn Nachrufe auf Reich-Ranicki auch über die Memorialisierung des Verstorbenen hinaus genützt werden, so stehen dabei weniger und, wie vielleicht zu erwarten wäre, methodische Überlegungen zur Literaturkritik, ihren Methoden (z.B. das literaturkritische Urteil und seine Objektivierbarkeit), ihrem Gegenstand (z.B. Poetik / literarische Ästhetik) und ihren theoretischen Grundlagen (z.B. ein suspekt gewordener Dualismus von Hoch- und Populärkultur), kurz: Fragen des aktuellen Literaturbetriebs im Zentrum; stattdessen werden über die Person des Kritikers und seine Vita Positionierungen innerhalb einer deutschen Erinnerungskultur festgelegt. Auch hier bewegen sich die Nachrufe mit historischen Opfer-Täter-Normierungen in vorgezeichneten und, aus Sicht aktueller Gedächtnisdiskurse, überholten Bahnen. Am Beispiel Reich-Ranickis als Vermittler zwischen kulturellen und national-religiösen Konstruktionen/ Positionen zeigt sich das Paradoxon von (populärer) Vermittlung, die ein Stück weit immer auch die Hierarchien und Grenzen reproduziert, gegen die anzutreten sie sich zum Ziel gesetzt hat. Nachrufen auf Reich-Ranicki, die selbst als populäre Texte gelten können und die über populäre Massenmedien zirkulieren, kommt für die Popularität Reich-Ranickis und seiner literaturkritischen, -verbreitenden und -vermittelnden Praxis sowohl retrospektive als auch prospektive Funktion zu: Indem sie diese in unterschiedlichen Bedeutungen und vom Faktum des Todes her rekonstruieren, für ein ,Jetzt' aktualisieren und für kommendes Gedenken veranschlagen, sichern sie Reich-Ranicki Popularität auch pro futuro - und zwar ungeachtet ihrer (negativen oder positiven) Valenz. 